\title{
Frequency, Clinical Manifestation and Treatment of Mitochondrial Epilepsy
}

\author{
Josef Finsterer $^{1 *}$ and Sinda Zarrouk-Mahjoub ${ }^{2}$
}

${ }^{1}$ Krankenanstalt Rudolfstiftung, Vienna, Austria

${ }^{2}$ Genetics Laboratory, Research Unit "Genetics Epidemiology and Molecular" Faculty of Medicine Tunis, Tunisia

\begin{abstract}
Epilepsy in mitochondrial disorders (MIDs, mitochondrial epilepsy) is an increasingly recognised topic, due to the increasing prevalence of mitochondrial disorders also in adult patients. Aim of the study was to describe mitochondrial epilepsy as a dominant or collateral feature of the phenotype in syndromic and non-syndromic MIDs. Syndromic MIDs obligatory associated with epilepsy include MELAS-syndrome, MERRF-syndrome, Leigh-syndrome, myoclonic epilepsy, myopathy and sensory ataxia (MEMSA)-syndrome, also known as spino-cerebellar ataxia with epilepsy (SCAE), ANS, and Alpers-Huttenlocher-disease. Occasionally, mitochondrial epilepsy occurs in LHON, NARP, MILS, MIDD, CPEO, KSS, IOSCA, or LBSL. All types of seizures occur in mitochondrial epilepsy but in adult patients most frequently generalized tonic-clonic seizures, partial seizures, myoclonic seizures, or epilepsia partialis continua occur. Treatment of mitochondrial epilepsy is not at variance from epilepsy due to other causes but mitochondrion-toxic antiepileptic drugs, such as valproic acid, carbamazepine, phenytoin, phenobarbital, and oxcarbazepine should be avoided. Generally well tolerated antiepileptic drugs in mitochondrial epilepsy include lamotrigine, levetirazetam, and lacosamide.
\end{abstract}

Keywords: Mitochondrial disorder; Epilepsy; Seizure; EEG, Central nervous system involvement; Antiepileptic drugs

\section{Introduction}

Epilepsy is one of the central nervous system (CNS) manifestations of syndromic as well as non-syndromic mitochondrial disorders (MIDs, mitochondrial epilepsy) [1]. Epilepsy in MIDs may be genetic without structural changes in the brain or due to structural/metabolic CNS changes secondary to the underlying mtDNA or nDNA mutation. Structural/metabolic causes of mitochondrial epilepsy include atherothrombotic or embolic ischemic stroke, stroke-like lesions, focal atrophy, white matter lesions, grey matter lesions, or cerebro-spinal fluid (CSF)-lactacidosis [2]. Little is known about type and prevalence of seizures and about treatment and outcome of epilepsy in MIDs. Aims of the study were to find out which types of epilepsy can be found in adult MID patients, how frequent is mitochondrial epilepsy, which antiepileptic drugs (AEDs) are the most effective, and which is the outcome of patients with mitochondrial epilepsy.

\section{Methods}

\section{Patients}

Retrospectively investigated were all patients with definite, probable, or possible MID in whom epilepsy was mentioned in their records. Patients with and without AED treatment were registered, but only adult patients were included.

\section{Diagnosing MIDs}

A MID was diagnosed as "definite" MID if the clinical manifestations suggested a MID and if there was biochemical or genetic evidence of a mitochondrial defect. A MID was diagnosed as "probable" MID if the clinical manifestations suggested a MID or if immune histological investigations of the muscle biopsy showed COX-negative fibers, ragged-red-fibers, SDH-hyper-reactive fibers, or abnormally shaped or structured mitochondria with or without para-crystalline inclusions or glycogen or fat depositions on ultra-structural investigations. A "possible" MID was diagnosed if the clinical Symptoms and signs suggestive of a MID were peripheral nervous system abnormalities, such as neuronopathy, neuropathy, or myopathy, CNS abnormalities such as impairment of consciousness or cognition, stroke-like episodes, stroke, headache, cerebellar signs, movement disorder, seizure or transverse syndrome, ocular abnormalities including visual impairment or double vision, ontological abnormalities such as sensory neural hearing loss, endocrine abnormalities, affecting the pituitary gland, the thyroid gland, the parathyroid gland, the pancreas, the adrenal gland, or the gonads, cardiac abnormalities due to arrhythmias or cardiomyopathy, or gastrointestinal or renal problems [3].

Instrumental findings suggestive of a MID included typical abnormalities on cerebral imaging, abnormal nerve conduction studies, needle electromyography, evoked potentials, ophthalmologic investigations indicative of cataract, glaucoma, or retinopathy, otological investigations indicating hypoacusis, blood tests showing elevated CK or liver function parameters, renal insufficiency, hyperlipidemia, hyperuricemia, anemia, thrombopenia, CSF tests showing elevation of lactate or protein, or pleocytosis, cardiologic investigations revealing arrhythmias or cardiomyopathy, gastrointestinal investigations showing steatosis, pancreatitis, cysts, diverticulosis, or colitis, or nephrological investigations revealing cysts, nephrolithiasis, or renal failure.

\section{Results}

Altogether, 60 patients with mitochondrial epilepsy were included. 3 with definite MID, 12 with probable MID, and 45 with possible MID. Two thirds of the patients were diagnosed with structural/metabolic epilepsy and one third with genetic epilepsy, according to the revised classification of the ILAE. The vast majority of the patients presented with generalized epilepsy and only a few patients with focal epilepsy. The history of status epileptics was positive in less than $10 \%$ of the patients.

*Corresponding author: Josef Finsterer, Krankenanstalt Rudolfstiftung, Postfach 20, 1180 Vienna, Austria, Tel: +43-1-71165-92085; Fax: +43-1-4781711; E-mail fifigs1@yahoo.de

Received March 12, 2014; Accepted March 24, 2014; Published March 27, 2014

Citation: Finsterer J, Zarrouk-Mahjoub S (2014) Frequency, Clinical Manifestation and Treatment of Mitochondrial Epilepsy. J Neurol Disord 2: 151. doi:10.4172/23296895.1000151

Copyright: (c) 2014 Finsterer J, et al. This is an open-access article distributed under the terms of the Creative Commons Attribution License, which permits unrestricted use, distribution, and reproduction in any medium, provided the original author and source are credited. 
Citation: Finsterer J, Zarrouk-Mahjoub S (2014) Frequency, Clinical Manifestation and Treatment of Mitochondrial Epilepsy. J Neurol Disord 2: 151. doi:10.4172/2329-6895.1000151

Page 2 of 2

Eighty percent of the patients were on AEDs at the time of the last visit. Reasons for being untreated were general objection against the AEDs, unauthorised discontinuation of the medication, or previous epilepsy, which had not recurred since years in the absence of AED treatment. Among those taking AEDs, $70 \%$ were on a monotherapy and $30 \%$ on a polytherapy with two or three other AEDs. The AEDs most frequently taken were levetirazetam, lamotrigine, valproic acid, and gabapentine.

\section{Discussion}

This study showed that in non-selected, adult patients mitochondrial epilepsy is most frequently due to structural or metabolic cerebral abnormalities and only rarely due to genetic causes. Most frequently patients with a MID present with generalized epilepsy and only rarely with focal seizures. AEDs applied to these patients were levetiracetam, lamotrigine, valproic acid, and gabapentine.

Epilepsy has been previously described as a phenotypic feature of a MID in $35-60 \%$ of the cases [4,5]. Epilepsy in syndromic MIDs most frequently occurs in mitochondrial encephalopathy, lactacidosis and stroke-like episodes (MELAS)-syndrome, myoclonic epilepsy with ragged-red fibers (MERRF)-syndrome, Leigh-syndrome, myoclonic epilepsy, myopathy and sensory ataxia (MEMSA)-syndrome, also known as spino-cerebellar ataxia with epilepsy (SCAE), ataxianeuropathy spectrum (ANS), which comprises mitochondrial recessive ataxia syndrome (MIRAS) and sensory ataxia with neuropathy, dysarthria and ophthalmoparesis (SANDO)-syndrome, or AlpersHuttenlocher-syndrome (AHS) [1]. An increasing number of patients with non-syndromic MIDs is recognized which also present with epilepsy as the dominant or as a collateral feature of the phenotype [6-8]. MIDs in these patients may be due to abnormal proteins of the respiratory chain or oxidative phosphorylation, disordered assembly of oxidative phosphorylation complexes, disordered mitochondrial translation, disorders of mt DNA maintenance, coenzyme-Q deficiency, or disordered mitochondrial import of proteins [2].

Interestingly, epilepsy phenotypes seem to be different in children and adults. While children frequently present with epilepsy syndromes, such as Otahara syndrome, West-syndrome, Lennox-Gastaut syndrome, Landau-Kleffner syndrome, or generalised and focal epilepsy, refractory neonatal status epilepticus, myoclonic epilepsy, infantile spasms, refractory/recurrent status epilepticus, or epilepsia partialis continua
$[9,10]$, adults seem to present more frequently with non-syndromic generalized epilepsy, as confirmed by the present investigation.

Concerning the antiepileptic treatment of mitochondrial epilepsy, mitochondrion-toxic AEDs should be avoided, whenever possible, and agents, that apparently have less negative effects on mitochondrial functions, such as lamotrigine, levetirazetam, and lacosamide, should be preferred.

\section{Conclusion}

Epilepsy in MID is most frequently due to structural or metabolic cerebral abnormalities. Mitochondrial epilepsy with normal cerebral imaging occurs in only a minority of the patients. In adult patients with mitochondrial epilepsy, generalised seizures are more frequent than focal seizures. AEDs presumably best tolerated in MIDs are levetirazetam, lamotrigine, and lacosamide. Outcome of MID patients with epilepsy is favourable if mitochondrion-toxic AEDs are avoided.

\section{References}

1. Rahman S (2012) Mitochondrial disease and epilepsy. Dev Med Child Neurol 54: 397-406.

2. Finsterer J, Zarrouk Mahjoub S (2012) Epilepsy in mitochondrial disorders. Seizure 21: 316-321.

3. Finsterer J, Jarius C, Eichberger $\mathrm{H}$ (2001) Phenotype variability in 130 adult patients with respiratory chain disorders. J Inherit Metab Dis 24: 560-576.

4. Debray FG, Lambert M, Chevalier I, Robitaille Y, Decarie JC, et al. (2007) Long-term outcome and clinical spectrum of 73 pediatric patients with mitochondrial diseases. Pediatrics 119: 722-733.

5. Khurana DS, Salganicoff L, Melvin JJ, Hobdell EF, Valencia I, et al. (2008) Epilepsy and respiratory chain defects in children with mitochondrial encephalopathies. Neuropediatrics 39: 8-13.

6. Schuelke M, Smeitink J, Mariman E, Loeffen J, Plecko B, et al. (1999) Mutant NDUFV1 subunit of mitochondrial complex I causes leukodystrophy and myoclonic epilepsy. Nat Genet 21: 260-261.

7. Barghuti F, Elian K, Gomori JM, Shaag A, Edvardson S, et al. (2008) The unique neuroradiology of complex I deficiency due to NDUFA12L defect. Mol Genet Metab 94: 78-82.

8. Saada A, Edvardson S, Rapoport M, Shaag A, Amry K, et al. (2008) C6ORF66 is an assembly factor of mitochondrial complex I. Am J Hum Genet 82: 32-38.

9. Lee YM, Kang HC, Lee JS, Kim SH, Kim EY, et al. (2008) Mitochondrial respiratory chain defects: underlying etiology in various epileptic conditions. Epilepsia 49: 685-690.

10. El Sabbagh S, Lebre AS, Bahi-Buisson N, Delonlay P, Soufflet C, et al. (2010) Epileptic phenotypes in children with respiratory chain disorders. Epilepsia 51: 1225-1235. 